\title{
Reactive Power Compensation on Egypt Electricity Network for Optimal Energy Saving
}

\author{
Youssef A. Mobarak \\ Department of Electrical and Computer Engineering, Faculty \\ of Engineering, King Abdulaziz University, Saudi Arabia, and \\ Electrical Engineering Department, Aswan University, Faculty \\ of Energy Engineering, Aswan, Egypt
}

\author{
A. El-Bahnasawy \\ Electrical Engineering Department, \\ Aswan University, \\ Faculty of Energy Engineering, \\ Aswan, Egypt
}

\author{
A. M. Hemeida \\ Electrical Engineering Department, \\ Aswan University, \\ Faculty of Energy Engineering, \\ Aswan, Egypt \\ Mohamed M. Hamada \\ Department of Electrical Engineering, \\ Minia University, \\ Faculty of Engineering, \\ Minia, Egypt
}

\begin{abstract}
This paper introduces the load flow on the Egypt Electricity Network system. The effect of capacitor compensation has been studied from three points. First, the problem of the series capacitor compensation was considered. The second type of compensation considered is shunt compensation. Finally, a mixed of shunt and series capacitors compensation was implemented. The analysis results were discussed based on the maximum reduction in the generated MVAR. The load variation is accounted for by considering three different load levels classified as light, medium and peak load with pre-specified durations. When solving the capacitor placement problem, the number, size, location and control settings of the capacitors at different load levels were determined. The load flow program is solved by the Power World Simulator (PWS) software. The results of series capacitor, shunt capacitor, and mixed compensation were studied. The investigation has been done for single capacitor and multi-capacitor compensation. Both $500 \mathrm{kV}$ and $220 \mathrm{kV}$ overhead lines have been considered.
\end{abstract}

Keywords-voltage; frequency; reactive power; load flow; Egyptian

\section{INTRODUCTION}

Egypt is the largest non-member of the Organization of the Petroleum Exporting Countries oil producer in Africa and the second-largest dry natural gas producer on the continent. Egypt is the largest oil and natural gas consumer in Africa, accounting for more than $20 \%$ of continent's total oil consumption and more than $40 \%$ of total dry natural gas consumption in 2013 [12]. Automatic generation control (AGC) is an important problem in power system operation and control. Whenever a small load perturbation occurs, it causes changes in tie-line power flow and frequency deviation. Many investigations in the area of AGC of interconnected power systems have been carried out in the past [3-5] and a number of control strategies have been proposed to improve the performance of AGC. Consequently, the non-linear nature of the load frequency control (LFC) problem makes it difficult to ensure stability for all operating points when an integral controller is used [6-7]. The application of adaptive control theory to the LFC problem eliminates some of the problems associated with classical and modern control [8-10]. Voltage instability has been observed in several forms, which was reached to complete blackouts of power systems in several countries [11]. Power system institutes such as IEEE, CIGRE, IEE and EPRI have turned great attention to the subject [12]. An IEEE subcommittee was formed in 1986 for its study [13-15]. In future, the subject will have a direct access to other interests such as power system security, reliability, planning, control methods and power system harmonics suppression. Electronic static VAR compensators, flexible AC transmission systems and HVDC systems will be widely used to counteract the effect of such devices and to improve the voltage stability situation of large systems [16-18]. Power quality is a recent subject that appeared with the complication of power systems [19-22]. Reactive power compensation is an important issue in electric power systems, involving aspects of service like operation, economy and quality. Consumer loads (residential, industrial etc.) impose active and reactive power demand, depending on their characteristics. Active power is converted into useful energy, like light or heat. Reactive power must be compensated to guarantee an efficient delivery of active power to loads, thus releasing system capacity, reducing system losses, and improving system power factor and bus voltage profile. Due to this, optimization of the capacitor placement problem (CPP) is important for studying the effect of capacitor compensation of a certain system. The problem of reactive power control for the Egypt Electricity Network (EEN) has been addressed in this paper.

\section{EEN DESCRIPTION AND DATA}

EEN operates a large and extensive transmission system consisting of six major subareas. These areas are Upper Egypt,

Corresponding author: Youssef A. Mobarak 
Cairo, Medium Delta, Canal, West Delta and Alexandria (Alex.). The peak load is about $21051 \mathrm{MW}$ and is increasing at an average rate of $6-7 \mathrm{MW}$ per year. The system consists of large steam units $(9698 \mathrm{MW})$ and many different sizes of gas turbines in about 10 major and several smaller power plants such as 937 hydraulic, 106.4MW w-level and 49.61MW wind turbine. EEN is shown in Figure 1 for single line diagram. The load flow solution has been carried out using PWS. The program solves the load flow problem using the NewtonRaphson method. The results of the peak, medium and light load levels solutions are presented and explained. The results were compared with actual load flow in the company file and were found identical.

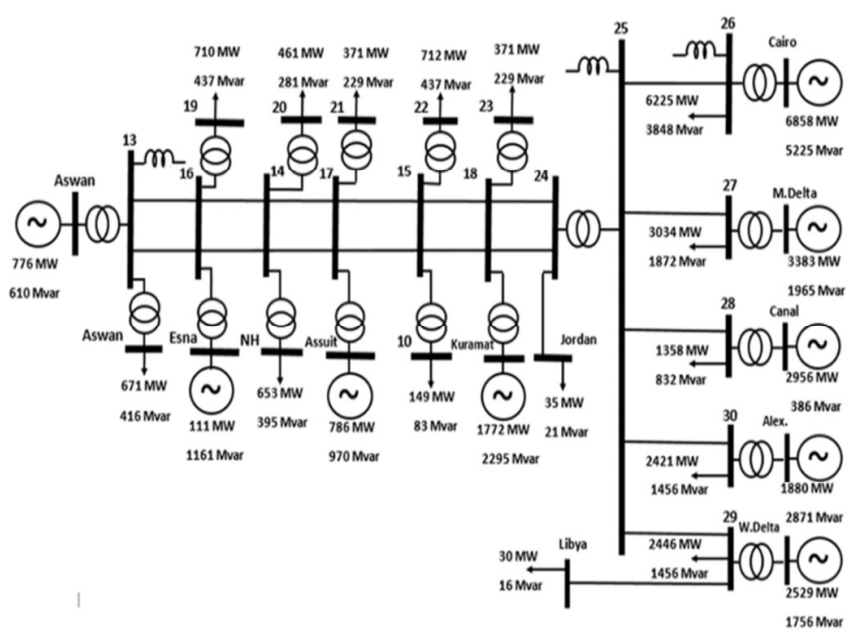

Fig. 1. Single line diagram of the studied EEN system.

In general, the total peak load for EEN was 19,677MW. The total power consumption in Cairo was the largest compared to the other cities at $6255 \mathrm{MW}$ which represented about $32 \%$ of the total area load. Upper Egypt network is considered the second largest load sink representing around $21 \%$ of total area load. Upper Egypt, Alexandria, Middle Delta, West Delta and Canal peak time power consumption is 4133MW, 2421MW, 3034MW, 2476MW, 1358MW respectively. Table I gives the active, reactive and complex power consumption of each area.

TABLE I. LOAD CONSUMPTION AT PEAK TIME

\begin{tabular}{|c|c|c|c|}
\hline Area & Active (MW) & Reactive (MVAR) & Complex (MVA) \\
\hline Upper Egypt & 4133 & 2528 & 4845 \\
\hline Cairo & 6255 & 3848 & 7344 \\
\hline Alexandria & 2421 & 1456 & 2825 \\
\hline M. Delta & 3034 & 1872 & 3565 \\
\hline W. Delta & 2476 & 1472 & 2881 \\
\hline Canal & 1358 & 832 & 1593 \\
\hline Total & $\mathbf{1 9 , 6 7 7}$ & $\mathbf{1 2 , 0 0 8}$ & $\mathbf{2 3 , 0 5 3}$ \\
\hline
\end{tabular}

\section{RESULTS AND DISCUSSION}

The load flow solution considered $500 / 220 \mathrm{kV}$ network at light, medium, and peak time. This represents a single line diagram of EEN in which all bus voltages, loads and flow lines are presented. The load flow solution of the 500/220kV EEN, shows that there are 31 transmission lines carrying power between the $500 / 220 \mathrm{kV}$ substations. The total generated active power from the stations, at peak time, was about $21051 \mathrm{MW}$. On the other hand, the reactive power generation at peak time was about 17239MVAR.

\section{A. Conventional Load Flow}

Figure 2 shows the voltage profile of all buses of the Egyptian 32-bus system as obtained from the load flow. It can be seen that all bus voltages are within the acceptable level $( \pm 7 \%$ ) except buses 24 to 32 , which have about 0.9 pu. The participating factor for this mode has been calculated and the result is shown in Figure 3. The result shows that, buses 11 (Naj-Hammadi), 19 (Qena) and 20 (Sohag) have the highest participation factors for the critical mode. The largest participation factor value $(0.2294)$ at bus 20 indicates the highest contribution of this bus to the voltage collapse.

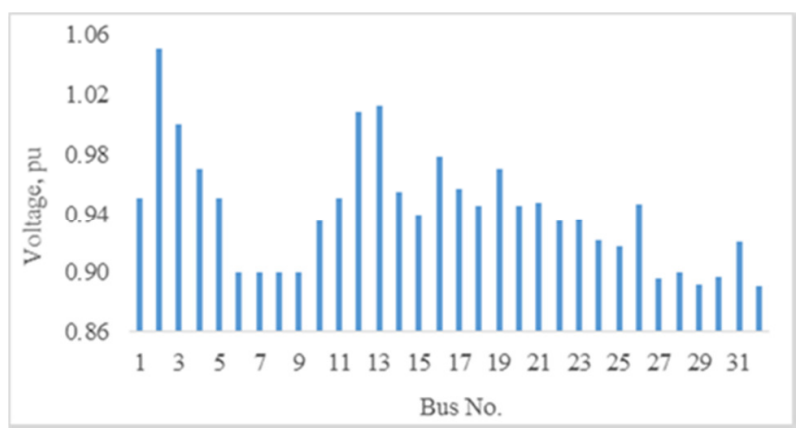

Fig. 2. Voltage profiles of all buses of the EENPS

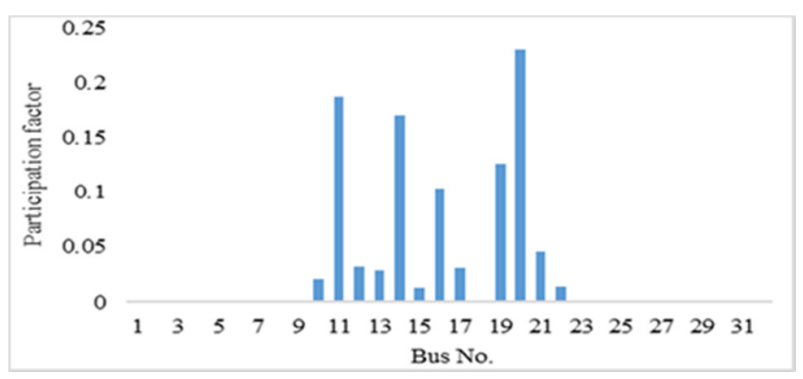

Fig. 3. Participating factor of all buses

\section{B. Load Flow Dependent on Voltage and Frequenccy}

Let us consider a nominal voltage conventional load flow as shown in Figure 4, when load is voltage dependent (the factors $\mathrm{k}_{\mathrm{pv}}$ and $\mathrm{k}_{\mathrm{qv}}$ are 2 and 2 respectively), and load flow when load is voltage and frequency dependent. Slack bus power is specified. The factors $\mathrm{k}_{\mathrm{pf}}$ and $\mathrm{k}_{\mathrm{qf}}$ are 2 and -2 respectively, and $\mathrm{P}_{\mathrm{G} 1}=16 \mathrm{pu}$. The solution for system frequency is such that loadgeneration-loss balance is achieved at that frequency. With the specifications given above, the total generation is greater than the load and losses at the nominal frequency. Therefore, frequency will be higher than the nominal frequency $(50.8494 \mathrm{~Hz})$. If $\mathrm{k}_{\mathrm{pf}}$ is changed to 3.0 , the frequency will be higher than the nominal frequency $(52.275 \mathrm{~Hz})$. 


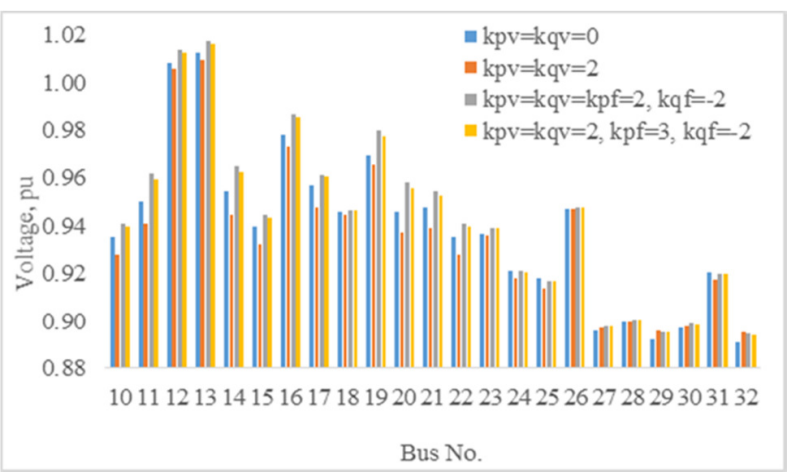

Fig. 4. Voltage profiles of all buses of the EENPS at different load models.

\section{Effect of Series Compensation on EEN}

In this section, the effect of series capacitor compensation on $500 / 220 \mathrm{kV}$ overhead lines is studied. Single, double and triple compensations were considered separately. Beyond triple capacitor compensation, the difference of benefits is not significant and hence we don't recommend the implementation of more than three series capacitors at a time. It has been found that the best location to install the single capacitor compensation will be in the transmission line connecting the $500 \mathrm{kV}$ substations number 14 and 16. As shown in Figure 5 we can get a reduction in total generated reactive power of 3671.9MVAR which is about 21.3 of the total generation during peak time. On the other hand, the generated active power will be increased by $112 \mathrm{MW}$ in this case. Regarding the double series capacitor compensation for 500KV network, the best locations in $500 \mathrm{kV}$ network will be in the lines connecting substations (14-16), and (15-17) which should compensate at $65 \%$ of line impedance. By implementing the above two series capacitors the total reduction in the total generated complex power will be around 4172MVAR, which is about $24.2 \%$ of the total generation, at peak time. On the other hand, the generated active power will be increased by $151 \mathrm{MW}$ in this case. Regarding the results of the triple series capacitor compensation for EEN, it was found that the best locations at peak time should be in the lines overhead line connecting substations (14-16), (15-17), and (18-24) which should compensate at $66 \%$ of line impedance.

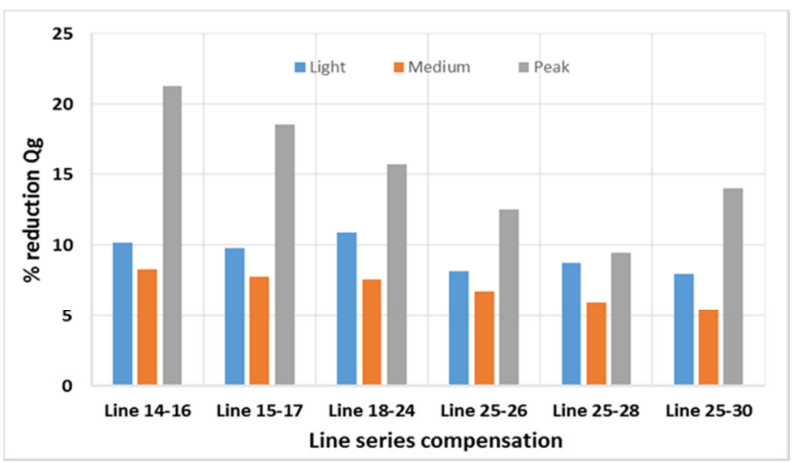

Fig. 5. The effect of single capacitor series compensation applied on EEN.

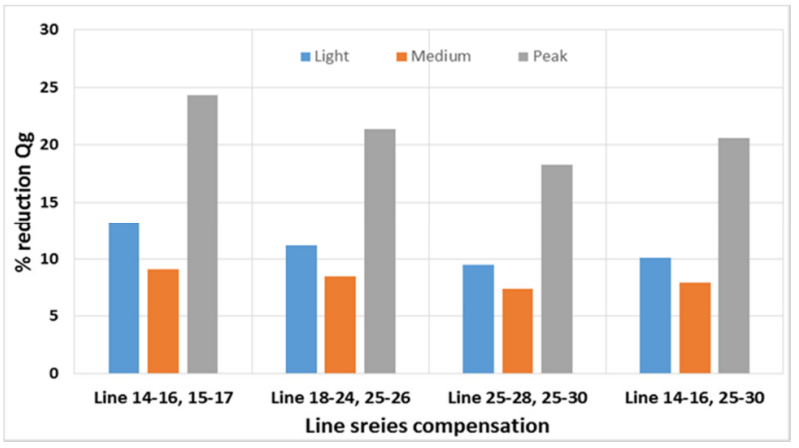

Fig. 6. The effect of double capacitor series compensation applied on EEN.

Installing these capacitors will reduce the total generated MVAR in EEN by around $25.5 \%$. The reduced reactive generated power is 4396MVAR at peak time. However, the generated active power will be increased by about $151 \mathrm{MW}$. The impact of triple compensation on medium and light load are shown in Figure 7. When series compensation was applied in the $500 / 220 \mathrm{kV}$ overhead lines, it was found that the best reduction in the generated MVAR was obtained after using triple compensation. The reduction was about $25.5 \%$ of total system generated MVAR at peak time. But generally, there was no big difference in the reduced MVAR when using single, double or triple series compensation. The reduction generated MVAR while using single, double or triple series compensation at system peak load was $3671.9,4172$, and 4396 respectively as shown in Figure 8.

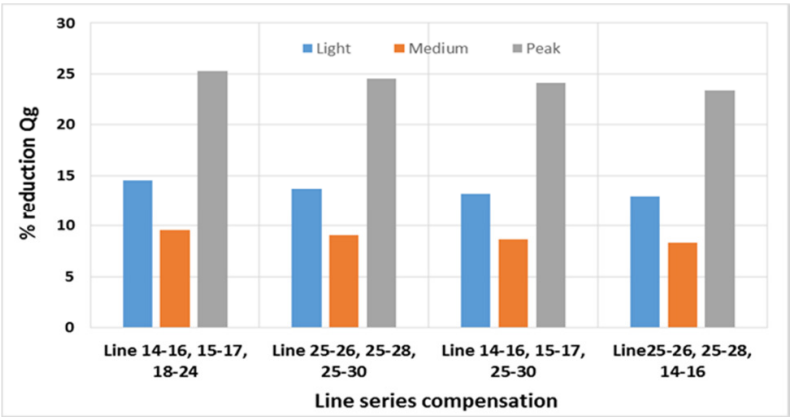

Fig. 7. The effect of triple capacitor series compensation applied on EEN.

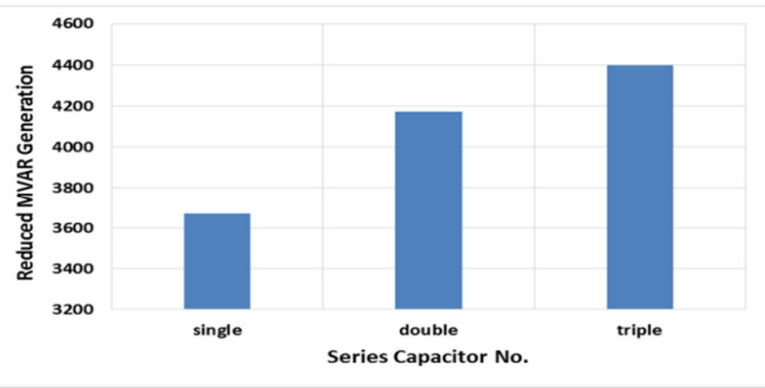

Fig. 8. Reduced generated MVAR of EEN series compensation. 


\section{Effect of Shunt Compensation on EEN}

Shunt capacitor compensation has been seen based on the hypothesis that EEN can reduce up to 9050MVAR. The reduction represents around $52.5 \%$ of total system generation. This is achievable only when the shunt capacitive units are installed at the right buses. Analysis has been conducted for single, double and triple capacitor compensation. It was seen that shunt compensation in more than three locations does not worth the effort.

In case of single-bus compensation, only around 5378MVAR can be saved in the generation at peak time. This reduction is about $31.2 \%$ of the system generation. The reactive power compensation should be installed at substation number 14. The rating at the capacitor bank should be 636MVAR (Figure 9). The compensation factor is $94 \%$ of bus load at this value. The value of the capacitive bank at peak medium and light loads is also given. On the other hand, the system generation can be declined by about 8688MVAR, which is almost $50.4 \%$ of system generation, by applying double-bus compensation. The shunt elements should be installed at buses 17 and 28 with 138 and 541MVAR respectively. The values of shunt compensation are presented for peak, medium and light periods in Figure 10. For 3-bus shunt compensation, the best reduction in the generated MVAR was almost 9050MVAR which is better than the 2-bus compensation case by around $326 \mathrm{MVAR}$. This reduction is about $52.5 \%$ of total generated MVAR. To achieve this reduction, three shunt elements should be installed at buses 28, 30 and 16 with rated values of 541, 437 and 153MVAR respectively (Figure 11). All peak, medium and light load compensation values are shown in Figure 11.

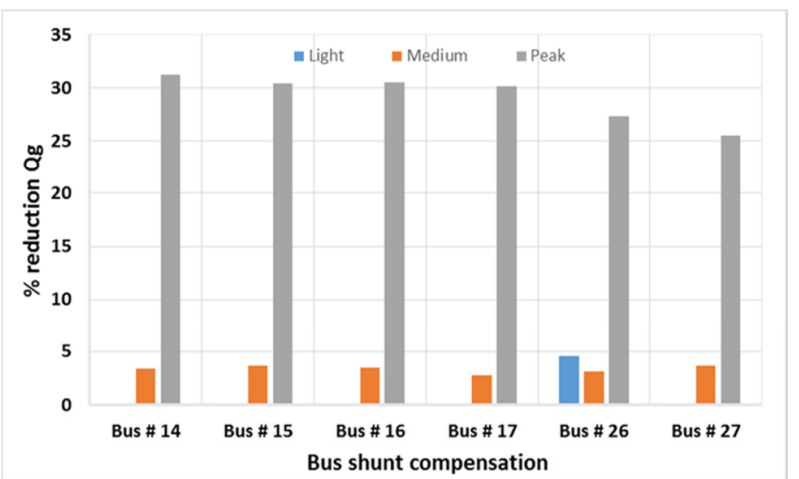

Fig. 9. The effect of single capacitor shunt compensation applied on EEN.

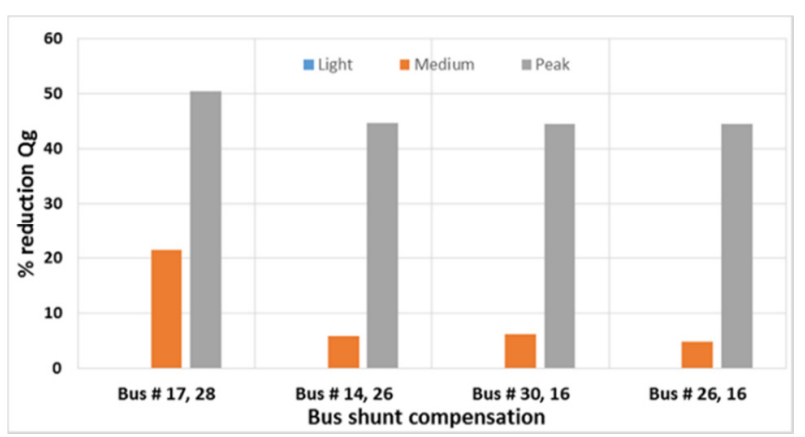

Fig. 10. The effect of double capacitor shunt compensation applied on EEN.

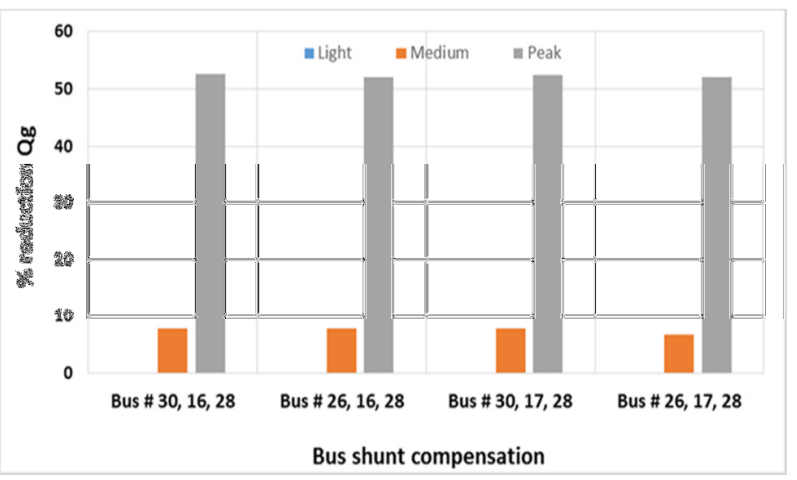

Fig. 11. The effect of triple capacitor shunt compensation applied on EEN.

Result comparison of shunt capacitor compensation has been done for each network based on the value of reduced MVAR of generation. For EEN, the maximum achievable reduction in the generated MVAR was 9050MVAR, which is $52.5 \%$ of total system generation, at triple capacitor compensation at peak time. Figure 12 compares single, double and triple compensation on EE network.

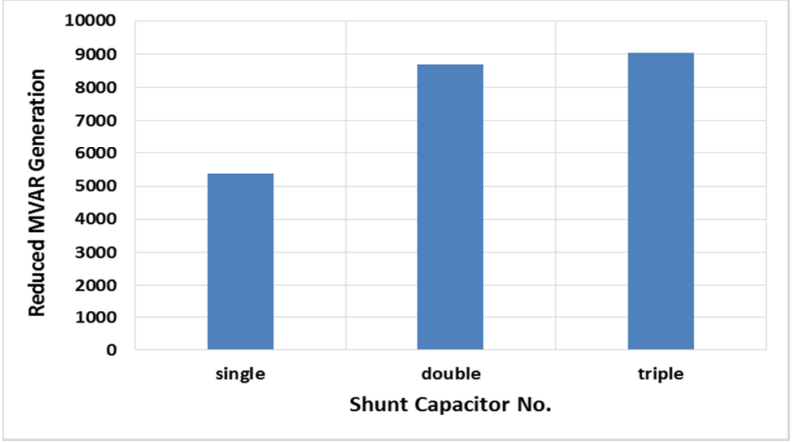

Fig. 12. Reduced MVAR generated of EEN shunt compensation.

\section{E. Effect of Mixed Compensation on EEN}

This section presents the results of mixed shunt and series capacitor compensation on 500/220kV network. Applying mixed capacitor compensation on EEN system results in a reduction of 7997MVAR of total system generation during peak time. This is representing about $46.39 \%$ of total system generated MVAR. Mixed compensation increases the total generated MW by almost $6 \mathrm{MW}$ during peak time. This testing was performed for single and double mixed capacitor compensation. In the case of single mixed compensation, maximum reduction in the generated MVAR obtained during peak load is 7130 MVAR which is about $41.36 \%$ of total generated MVAR. The single shunt compensation should be added on bus number 14 with a size of 298MVAR. On the other hand, single series compensation should be added into the $380 \mathrm{kV}$ transmission connecting substations (16-14). The compensation rate of the line should be 65 of line impedance. Figure 13 shows the results of single mixed compensation on EEN system. In this Figure, the best locations are sorted based on maximum reduction of the generated MVAR. Compensation values of all three load levels (peak, medium and light) are also presented. 


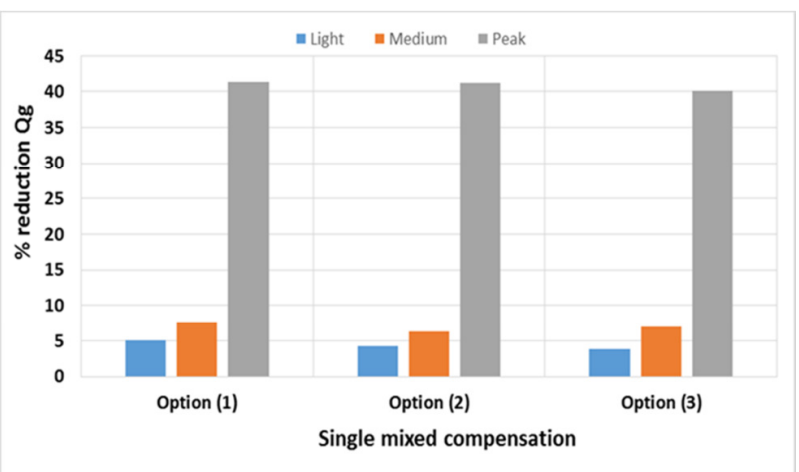

Fig. 13. The effect of single mixed compensation applied on EEN.

In the case of double mixed compensation, total reduction of 7997MVAR of total generation was achieved. This is about $46.4 \%$ of the system generation. The two shunt elements to be added are on buses 14 and 17 with values of 602 and 67MVAR respectively. On the other hand, two $500 \mathrm{kV}$ lines, connecting substations (14-16) and (17-15), should be compensated by series capacitive elements at $70 \%$ and $65 \%$ of the investigation results of double-mixed compensation case on EEN (Figure 14). The best locations are based on maximum reduced generated MVAR, and compensation values of all three load levels (peak, medium and light) are presented. The results of the mixed shunt and series capacitor compensation were compared for each network and bench marked. The comparison has been done for each network based on the value of reduced generation MVAR. Another results comparison is to compare the increased MW in the generation.

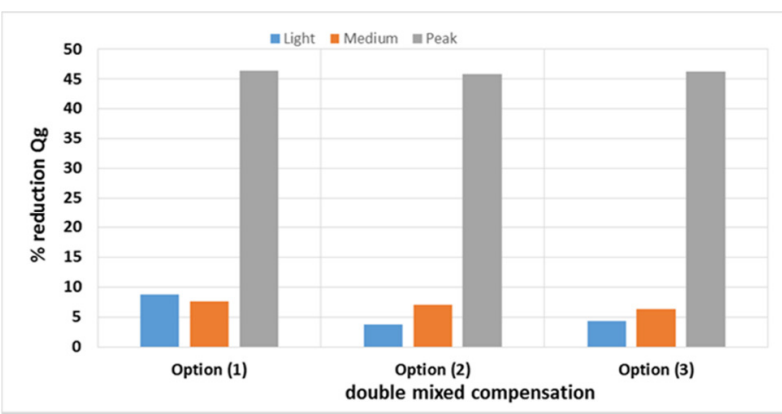

Fig. 14. The effect of double mixed compensation applied on EEN.

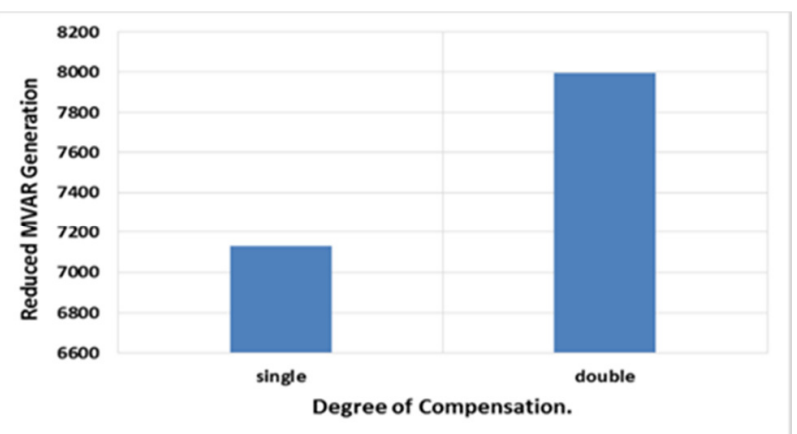

Fig. 15. Reduced generated MVAR of EEN mixed compensation.
For the $500 \mathrm{kV}$ network, the maximum achievable reduction in the generated MVAR was at double capacitor compensation at $930.6 \mathrm{MVAR}$, which is $46.4 \%$ of total system generation, at peak time. Figure 15 compares single and double compensation on the $500 \mathrm{kV}$ network. Prior to capacitor installation, a load flow study was run to obtain the present EEN system condition presented in Table II which shows the generated active power, generated reactive power, generated complex power, system KW losses, the cost of energy losses during peak, medium and light load levels and the cost of system energy losses.

TABLE II. SYSTEM CONDITIONS BEFORE CAPACITOR PLACEMENT

\begin{tabular}{|c|c|c|c|}
\hline \multirow{2}{*}{} & \multicolumn{3}{|c|}{ Load Condition } \\
\cline { 2 - 4 } & Light & Medium & Peak \\
\hline Total generated active power P (MW) & 19567 & 18335 & 21051 \\
\hline Total generated reactive power Q (MVAR) & 18284 & 15057 & 17239 \\
\hline Total generated complex power S (MVA) & 26780 & 23725 & 27209 \\
\hline Real power losses (MW) & 7088 & 2441 & 1373 \\
\hline Cost of Energy Losses (\$) & 931,363 & 641,495 & 180,412 \\
\hline Total Cost of Energy Losses (\$/year) & \multicolumn{3}{|c|}{$\mathbf{2 , 0 4 3 , 1 3 8}$} \\
\hline
\end{tabular}

\section{CONCLUSION}

The installation of series, shunt or mixed capacitors reduced energy losses in the system. The maximum obtained reduction in generated MVARs was $25.5 \%, 52.5 \%$ and $46.6 \%$ for the $500 / 220 \mathrm{kV}$ network. Shunt compensation at substations with large loads results in a better reduction of the generated MVA than at substations with low loads. The reduced MVA of shunt compensation is more than the one of mixed or series compensation.

\section{REFERENCES}

[1] A. Alshehri, A. Hussain, Y. A. Mobarak, "Energy-conversion measures in the industries of Saudi Arabia and development of methodology for certification of energy personnel in the kingdom", Energy Policy, Vol. 64, No. 1, pp. 203-208, 2014

[2] Y. A. Mobarak, "Voltage collapse prediction for Egyptian interconnected electrical grid EIEG”, International Journal on Electrical Engineering and Informatics, Vol. 7, No. 1, pp. 79-88, 2015

[3] M. Al-Amin Sarker, A. K. M. Kamrul Hasan, "Load Frequency Control in Power System", SEU Journal of Science and Engineering, Vol. 10, No. 2, pp. 24-30, 2016

[4] K. S. S. Ramakrishna, P. Sharma, T. S. Bhatti, "Automatic generation control of interconnected power system with diverse sources of power generation", International Journal of Engineering, Science and Technology, Vol. 2, No. 10, pp. 51-65, 2010

[5] R. S. Shekhawat, S. Garg, "Automatic Generation Control-An Enhanced Review", International Journal of Engineering Trends and Technology, Vol. 5, No. 2, pp. 75-84, 2017

[6] A. Delassi, S. Arif, L. Mokrani, "Load frequency control problem in interconnected power systems using robust fractional PID controller", Ain Shams Engineering Journal, Vol. 9, No. 1, pp. 77-88, 2018

[7] R. Umrao1, S. Kumar, M. Mohan, D.K.Chaturvedi, "Load Frequency Control Methodologies for Power System", 2nd International Conference on Power, Control and Embedded Systems, Allahabad, India, December 17-19, 2012

[8] Y. L. Abdel-Magid, M. A. Abido, "AGC tuning of interconnected reheat thermal systems with particle swarm optimization", 10th IEEE International Conference on Electronics, Circuits and Systems, Sharjah, United Arab Emirates, December 14-17, 2003

[9] D. Rerkpreedapong, A. Hasanovic, A. Feliachi, "Robust load frequency control using genetic algorithms and linear matrix inequalities", IEEE Transactions on Power Systems, Vol. 18, No. 2, pp. 855-861, 2003 
[10] C. S.Chang, F. U. Weihui, "Area load frequency control using fuzzy gain scheduling of PI controllers", Electric Power Systems Research, Vol. 42, pp. 145-152, 1997

[11] P. Nagendra, T. Datta, S. Halder, S. Paul, "Power system voltage stability assessment using network equivalents - A review", Journal of Applied Sciences, Vol. 10, pp. 2147-2153, 2010

[12] A. Dhamanda, A. K. Bhardwaj, "Automatic generation control of thermal generating unit by using conventional and intelligent controller", International Journal of Electrical Engineering \& Technology, Vol. 5, No. 10 , pp. 56-64, 2014

[13] G. A. Chown, R. C. Hartman, "Design and experiences with a FLC for automatic generation control AGC", IEEE Transactions on Power Systems, Vol. 13, No. 3, pp. 965-970, 1998

[14] Y. A. Mobarak, A. M. Elnoby, "Improvement of Dynamic Performance of Interconnected Power Systems with Static Synchronous Series Compensator", International Journal of Electrical and Electronics Engineering, Vol. 7, No. 2, pp. 21-30, 2015

[15] Y. A. Mobarak, S. A. Deraz, M. El-Shahat Dessouki, R. Almazmomi, "Optimal Control Techniques in Applicable Values of Turbine Speed Governor Regulation", SSRG International Journal of Electrical and Electronics Engineering, Vol. 2, No. 8, pp. 24-35, 2015

[16] A. M. Elnoby, G. El-Saady, Y. Mobarak, M. A. A. Wahab, M. M. Hamada, M. A. Abdel-Moamen, "Robust Technique LFC of Two-Area Power System with Dynamic Performance of Combined SMES and SSSC Control", International Journal of Advances in Engineering \& Technology, Vol. 8, No. 2, pp. 46-58, 2015

[17] Y. L. Karnavas, D. P. Papadopoulos, "AGC for autonomous power system using combined intelligent techniques", Electrical Power Systems Research, Vol. 62. No. 3, pp. 225-239, 2002

[18] K. Sabahil, M. A. Nekouil, M. Teshnehlab, M. Aliyari, M. Mansouri, "Load frequency control in interconnected power system using dynamic modified neural networks", 2007 Mediterranean Conference on Control \& Automation, Athens, Greece, June 27-29, 2007

[19] G. Shabib, Y. A. Mobarak, M. H. El-Ahmar, "Combined SVC and SSSC controllers for power system transient stability improvement", 13th International Middle East Power Systems Conference, Assiut, Egypt, December 21-23, 2009

[20] Y. A. Mobarak, M. M. Hussein, "Voltage instability of initiation fault duration as influenced by nodes short circuit levels NSCL", International Journal of Electrical and Computer Engineering, Vol. 6, No. 3, pp. 1305 13018,2016

[21] Y. A. Mobarak, "Fault duration for voltage instability and voltage collapse initiation as influenced by generator voltage magnitudes GVM", Journal of Engineering Sciences, Vol. 40, No. 3, pp. 846-866, 2012

[22] Y. A. Mobarak, "Effects of the Droop Speed Governor and Automatic Generation Control AGC on Generator Load Sharing of Power System", International Journal of Applied Power Engineering, Vol. 4, No. 3, pp. 21-30, 2015

\section{AUTHORS PROFILE}

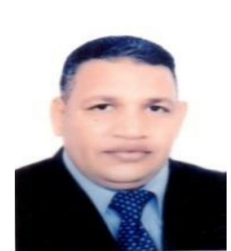

Youssef A. Mobarak was born in Egypt. He received his B.Sc. and M.Sc. degrees in Electrical Engineering from South Valley University, Aswan, Egypt, in 1997 and 2001 respectively and his Ph.D. from Cairo University, Egypt, in 2005. He joined the Electrical Engineering Department, Faculty of Energy Engineering, Aswan University as a Demonstrator, Assistant Lecturer, and Assistant Professor during the periods of 1998-2001, 2001-2005, and 2005-2013 respectively. In 2014 he obtained the Associate Professor rank. He joined Artificial Complex Systems, Hiroshima University, Japan as a researcher in 2007-2008. Also, he joined King Abdulaziz University, Rabigh, Faculty of Engineering 2010 to present. His research interests are power system planning, operation, and optimization techniques applied to power systems, nanotechnology materials via addition of nano-scale particles and additives for usage in industrial field.

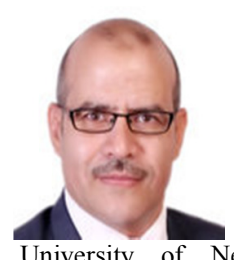

A. M. Hemeida was born in El-Menia, Egypt, on October, 21, 1968. He obtained his B.Sc., and M.Sc. in Electrical Power Engineering from Faculty of Engineering, Minia University, Minia, Egypt in 1992 and 1996 respectively. In 1992, he joined the Higher Institute of Energy, Aswan as Teaching Assistant. From 1998 to $1999 \mathrm{He}$ was a full time Ph.D. student in the Electrical and Computer Engineering Department, University of New Electrical Engineering Department, Faculty of Engineering, Assiut University, Assiut Egypt. From Oct. 2000 till August 2005 he was Assistant Professor at the Electrical Engineering Department, Higher Institute of Energy, South Valley University, Egypt. In Nov. 2005 he obtained Associate Professor rank. From Sept. 2006 until April, 2010 he was the chairman of Computer Science Department, Arrass Faculty of Science and Arts, Qassim University, Saudi Arabia. His research interests include power systems operation and control, artificial intelligence application in power systems, FACTS applications, voltage stability, and advanced control techniques in power systems

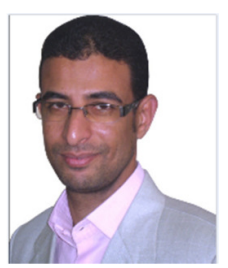

A. El-Bahnasawy was born in Egypt. He received his B.Sc. and M.Sc degrees in Electrical Engineering from South Valley University, Aswan, Egypt, in 2004 and 2010 respectively. He joined the Electrical Engineering Department, High Institute of Energy, South Valley University as a Demonstrator and Assistant Lecturer, during 2005-2010. He is a $\mathrm{PhD}$ student in Minia University from 2013.

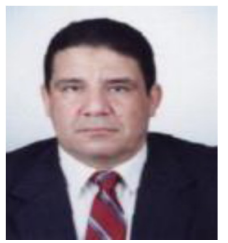

Mohamed M. Hamada was born in 1951. He received his B.Sc. and M.Sc. from Assiut University, Egypt in 1974 and 1982, respectively and Ph.D. from Manchester University Institute of Science and Technology (UMIST), UK, all in Electrical Engineering. From 1975 to 1983 he worked as a teaching assistant at Minia University, Egypt. From 1983 to 1989 he was with UMIST working with IPSA group and studying for $\mathrm{Ph}$.D. Since 1989, he has been a staff member at the Department of Electrical Engineering, Minia University. His main fields of interest are harmonics in power systems, HVDC, high voltage phenomena, voltage stability. 